OPEN ACCESS

Edited by: Elaine M. Bignell, University of Manchester, UK

Reviewed by: Donna MacCallum, University of Aberdeen, UK Malcolm Whiteway, Concordia University, Canada

${ }^{*}$ Correspondence: Alix T. Coste alix.coste@chuv.ch

Specialty section This article was submitted to Fungi and Their Interactions,

a section of the journal

Frontiers in Microbiology

Received: 06 January 2017 Accepted: 01 May 2017 Published: 16 May 2017

Citation:

Pierrehumbert A, Ischer F and Coste AT (2017) Unexpected Transcripts in Tn7 orf19.2646 C. albicans Mutant Lead to Low Fungal

Burden Phenotype In vivo. Front. Microbiol. 8:873

doi: 10.3389/fmicb.2017.00873

\section{Unexpected Transcripts in Tn7 orf19.2646 C. albicans Mutant Lead to Low Fungal Burden Phenotype In vivo}

\author{
Aude Pierrehumbert, Françoise Ischer and Alix T. Coste* \\ Institute of Microbiology, University of Lausanne and University Hospital Center, Lausanne, Switzerland
}

The commensal fungus Candida albicans is the major cause of fungal systemic infection in immuno-compromised patients, with a mortality rate approaching 50\% in the case of bloodstream infections. There is therefore a clear need to better understand fungal biology during infection to improve treatment. One of the particularities of $C$. albicans is its capacity to adapt to drastically diverse environments such as brain, bloodstream or gut. Adaptations to environmental change are mediated by transcription factors (TF) that modulate the expression of their target genes. Previous screening of a collection of Tn7 C. albicans TF mutants in vivo identified orf19.2646 as playing a crucial role in the ability of the fungus to survive within its host. Indeed, the orf19.2646 Tn7 interruption mutant strain displayed a reduced fungal burden compared to the wild-type strain. Surprisingly, an independent deletion mutant did not recapitulate the phenotype of the Tn7 interruption mutant. In the present study, we therefore investigated the difference between these two mutants and determined by performing a RACE analysis whether unexpected transcripts of the Tn7 mutant occurred. We found that two such transcripts upstream and downstream of the $\mathrm{Tn} 7$ insertion site were produced. The two transcripts were expressed in an orf19.2646 deletion mutant which displayed a significantly reduced fungal burden level compared to the wild-type in G. mellonella. When the regions corresponding to these transcripts were deleted in the Tn7 mutants, the strains lacking both regions displayed a fungal burden similar to that of the wild-type strain. This study shows for the first time that mRNA transcription may occur downstream of a $\operatorname{Tn} 7$ sequence. In addition, these results demonstrated that the low fungal burden phenotype observed in the orf19.2646 Tn7 mutant is due to the presence of these two transcripts together participating to an unidentified virulence mechanism to be further elucidated.

Keywords: Candida albicans, Tn7 mutant, unexpected transcript, virulence, fungal burden

\section{INTRODUCTION}

Candida albicans is one of the most successful fungal pathogens and is responsible for more than 50\% of all Candida spp. infections (Wisplinghoff et al., 2014). Candida infections are opportunistic infections occurring in immunosuppressed patients or patients with risk factors such as invasive surgery (patients in Intensive Care Units), broad spectrum antibiotherapy, or the use of catheters. 
Even if such patients are treated with antifungal drugs either prophylactically or because of an established infection, once the infection reaches the bloodstream and becomes systemic, prognosis is poor, with a mortality rate of up to $50 \%$ (McNeil et al., 2001; Gudlaugsson et al., 2003; Lortholary et al., 2014; Puig-Asensio et al., 2014). Even though crucial virulence factors have already been identified, such as filamentation (Braun and Johnson, 2000; Klein and Tebbets, 2007; Fuchs et al., 2010), biofilm formation (Harriott and Noverr, 2011; Akers et al., 2015; Nobile and Johnson, 2015; Rajendran et al., 2016), and iron level adaptation (Chen et al., 2011; Chen and Noble, 2012; Noble, 2013), a further understanding of the fungal factors necessary to successfully infect the host is urgently needed. For this reason, we have previously assessed the role of $C$. albicans transcription factors (TFs) in the mouse bloodstream and $G$. mellonella infection models, using a collection of around $300 \mathrm{TF}$ mutants (Vandeputte et al., 2011; Amorim-Vaz et al., 2015). This collection was achieved using a genomic library transposed with a Tn7 transposon flanked by a UAU cassette (Nobile and Mitchell, 2009). All plasmids thus obtained were sequenced at The Institute for Genomic Research, Rockville, MD (TIGR) consortium to determine the site of $\operatorname{Tn} 7$ insertion, and then used to generated C. albicans mutants (Nobile and Mitchell, 2009).

We initially focused our efforts on the Zn2Cys6 TF family (Vandeputte et al., 2011). Around 80 mutants were screened in a murine disseminated infection model. Groups of mice were infected with pools of 10 barcoded strains, consisting of 8 mutants, plus one isogenic wild-type strain and one avirulent isogenic cmpl mutant as controls. The relative proportion of mutants was measured by quantitative PCR (qPCR). This screening revealed that different strains displayed either hypoor hyper-kidney fungal burden phenotypes as compared to the wild-type strain (Vandeputte et al., 2011). In such pools of strains, the competitive fitness of strainsplays a role in the overall virulence. Strains showing a significantly reduced or increased fungal burden were then tested again in single strain infections to eliminate this "pool effect" (Vandeputte et al., 2011). Finally, 3 mutants were found to display a significantly reduced fungal burden in the murine kidney as compared to the wild-type strain: orf19.2646 (ZCF13), orf19.3405 (ZCF18), and orf19.3753 (SEF1). One of these mutants, orf19.2646 was of particular interest since it exhibited no growth deficiency in vitro and was not previously described (Vandeputte et al., 2011). To validate the observed phenotype, a revertant strain of the orf19.2646 Tn7 insertion mutant was also constructed. The re-introduction of a wildtype allele abolished the low fungal burden phenotype, thus confirming the role of orf19.2646 in this phenotype (Vandeputte et al., 2011). The low fungal burden phenotype was confirmed in our subsequent study in Galleria mellonella single strain infections (Amorim-Vaz et al., 2015). One caveat when using the Tn7-UAU cassette in a given gene is that the deduced ORF is interrupted and not deleted. In addition, the URA3 and ARG4 auxotrophic markers of the cassette are ectopically expressed. Indeed, auxotrophic markers such as URA3 have been shown to play a role in virulence (Brand et al., 2004). An independent mutant for orf19.2646 was therefore produced by complete deletion of the gene using a SAT1-flipper recyclable cassette, leading to the removal of the selection marker from the genome (Amorim-Vaz et al., 2015). This mutant was assessed by kidney fungal burden in mice and fungal burden larvae in $G$. mellonella. Surprisingly, this null mutant showed no significant reduced tissue colonization as compared to a wild-type strain (Amorim-Vaz et al., 2015). Taken together, these data suggest that the reduced kidney fungal burden phenotype of the mutant was dependent on the gene inactivation approach taken, either by Tn7 insertion or deletion, suggesting that some interfering factors played an additional role. It has been previously shown that chimeric transcripts can be produced upon transposon insertion in filamentous fungal species, such as Magnaporthe grisea and Mycosphaerella graminicola (Lo et al., 2003). These transcripts were described as containing a part of the Tn7L or Tn7R. Indeed, stop codons are present in all frames of Tn7L or Tn7R, thus causing premature polyadenylation of the transcript located upstream of the mutated ORF. We therefore addressed whether chimeric orf19.2646 transcripts could be produced in the Tn7-interrupted mutant and whether they could be responsible for the low fungal burden phenotype of the Tn7 insertion mutant.

\section{MATERIALS AND METHODS}

\section{Strains, Plasmids, and Growth Conditions}

Escherichia coli $\mathrm{DH} 5 \alpha$ was used as a host for plasmid propagation. To grow $\mathrm{DH} 5 \alpha$, Luria-Bertani (LB) broth medium was used and when necessary, supplemented with ampicillin $(0.1 \mathrm{mg} / \mathrm{ml})$. Cultures of $E$. coli were incubated at $37^{\circ} \mathrm{C}$ under constant agitation $(220 \mathrm{rpm})$ for $16-20 \mathrm{~h}$. For plate cultures, $0.7 \%$ BactoTM Agar (Brunschwig, Switzerland) was added.

All C. albicans strains used in this study are listed in Table 1. C. albicans were grown in complete Yeast Extract Peptone Dextrose (YEPD) medium ( $1 \%$ Bacto peptone, $0.5 \%$ yeast extract and $2 \%$ glucose) at $30^{\circ} \mathrm{C}$ under constant agitation (220 rpm). For growth on plates, 2\% BactoTM Agar was added to the medium. Yeast cells were transformed by a lithium-acetate procedure with slight modifications as previously described (Sanglard et al., 1996). After addition of Salmon sperm DNA ( $0.137 \mathrm{mg} / \mathrm{ml}$, Invitrogen) and heat shock steps, nourseothricin-resistant transformants were resuspended in $1 \mathrm{ml}$ of YEPD medium and incubated for at least $4 \mathrm{~h}$ at room temperature. The cells were harvested in $100 \mu \mathrm{l}$ of YEPD and plated on YEPD agar plates containing $200 \mu \mathrm{g} / \mathrm{ml}$ nourseothricin (Werner Bioagents, Germany).

All plasmids and primers used in this study are listed in Tables 2, 3, respectively. For pAC339, the first 885 bp of orf19.2646 was amplified and 1,000 bp upstream of the ATG using primers 2646-promF-KPN and the 2646-insert2xho (Table 3). This PCR product was cloned in pAC245 (Table 2) between KpnI and XhoI sites replacing the 1,000 bp upstream sequence by the truncated version of orf19.2646 flanked by $1,000 \mathrm{bp}$ upstream sequence. This construct was then transformed after a digestion by KpnI-SacI in ACY315 $(\operatorname{orf19} .2646 \Delta / \Delta)$ leading to strain ACY339. For the construction of pAP3, a PCR was performed using primers 2646-promFKPN and 2646-postRACE5'Reverse (Table 3) on the genomic DNA of ACY294 to amplify the region corresponding to the 
TABLE 1 | List of strains.

\begin{tabular}{|c|c|c|c|c|}
\hline Strain & Name in the text & Genotype & Genetic background & References \\
\hline ACY293 & WT collection & BWP17 ACT1::pDS1551 & $\mathrm{BCY} 31$ & Vandeputte et al., 2011 \\
\hline ACY294 & Tn7 mutant & $\begin{array}{l}\text { orf19.2646::Tn7-UAU, ACT1::pDS1551 } \\
\text { orf19.2646::Tn7-URA3 }\end{array}$ & BCY152 & Vandeputte et al., 2011 \\
\hline 点 $\quad$ ACY292 & WT revertant & orf19.2646::Tn7-UAU, ACT1::pAC249 orf19.2646::Tn7-URA3 & BCY152 & Vandeputte et al., 2011 \\
\hline 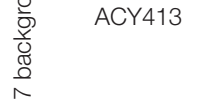 & & $\begin{array}{l}\text { orf19.2646::Tn7; } \operatorname{Tn} 7 \text { upstream; } \operatorname{Tn} 7 \text { downstream::(pAC321) } \\
\text { FRT-SAT1-FRT/... } \\
\text { orf19.2646:: } T n 7 ; \operatorname{Tn} 7 \text { upstream; } \operatorname{Tn} 7 \text { downstream }\end{array}$ & BCY152 & This study \\
\hline ACY417 & & $\begin{array}{l}\text { orf19.2646::Tn7; } \operatorname{Tn} 7 \text { upstream; } \operatorname{Tn} 7 \text { downstream::FRT/ } \\
\text { orf19.2646::Tn7; } \operatorname{Tn} 7 \text { upstream; } \operatorname{Tn} 7 \text { downstream }\end{array}$ & ACY413 & This study \\
\hline ACY420 & & $\begin{array}{l}\text { orf19.2646::Tn7; } \operatorname{Tn} 7 \text { upstream::(pAC323) FRT-SAT1-FRT; } \\
\text { Tn7downstream::FRT/ } \\
\text { orf19.2646::Tn7; } \operatorname{Tn} 7 \text { upstream; } \operatorname{Tn} 7 \text { downstream }\end{array}$ & ACY417 & This study \\
\hline ACY421 & & $\begin{array}{l}\text { orf19.2646::Tn7; } \operatorname{Tn} 7 \text { upstream::FRT; Tn7downstream::FRT/ } \\
\text { orf19.2646::Tn7; Tn7 upstream; } \operatorname{Tn} 7 \text { downstream }\end{array}$ & ACY420 & This study \\
\hline ACY423 & & $\begin{array}{l}\text { orf19.2646::Tn7; Tn7 upstream::FRT; Tn7downstream::FRT/ } \\
\text { orf19.2646::Tn7; Tn7 upstream::FRT; Tn7downstream }\end{array}$ & ACY423 & This study \\
\hline ACY428 & Up + Do deletion & $\begin{array}{l}\text { orf19.2646::Tn7; Tn7 upstream::FRT; Tn7downstream::FRT/ } \\
\text { orf19.2646::Tn7; Tn7 upstream:::FRT; } \\
\text { Tn7downstream::(pAC321) FRT-SAT1-FRT/... }\end{array}$ & ACY423 & This study \\
\hline ACY430 & Do deletion & $\begin{array}{l}\text { orf19.2646::Tn7; Tn7 upstream; Tn7downstream::FRT/ } \\
\text { orf19.2646::Tn7; Tn7 upstream; Tn7downstream::(pAC321) } \\
\text { FRT-SAT1-FRT/... }\end{array}$ & ACY417 & This study \\
\hline
\end{tabular}

\begin{tabular}{|c|c|c|c|c|c|}
\hline \multirow{10}{*}{ 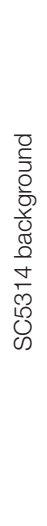 } & \multicolumn{5}{|l|}{ SC5314 } \\
\hline & ACY297 & & orf19.2646/orf19.2646 $\Delta$ & SC5314 & Amorim-Vaz et al., 2015 \\
\hline & ACY315 & Deletion mutant & orf19.2646 $\Delta / \Delta$ & SC5314 & Amorim-Vaz et al., 2015 \\
\hline & ACY339 & S-revertant & orf19.2646 $\Delta$ /orf19.2646 $\Delta \because:(\mathrm{pAC339)}$ FRT-SAT1-FRT & ACY315 & This study \\
\hline & ACY352 & & orf19.2646 $\Delta$ /orf19.2646 $\Delta::$ pAC339 FRT & ACY339 & This study \\
\hline & ACY362 & $\mathrm{S}+\mathrm{WT}$ revertant & $\begin{array}{l}\text { orf19.2646 } \Delta \because:(\mathrm{pAC} 253) \text { FRT-SAT1-FRT/orf19.2646 } \\
\Delta \because:(\mathrm{pAC339)} \text { FRT }\end{array}$ & ACY352 & This study \\
\hline & ACY402 & Up-revertant & orf19.2646 $\Delta$ /orf19.2646 $\Delta::$ pAP3 & ACY315 & This study \\
\hline & ACY403 & & orf19.2646/orf19.2646::pAP4 & SC5314 & This study \\
\hline & ACY404 & Do-revertant & orf19.2646 $\Delta$ /orf19.2646::pAP4 & ACY297 & This study \\
\hline & ACY405 & Up + Do-revertant & orf19.2646::pAP3/orf19.2646::pAP4 & ACY401 & This study \\
\hline
\end{tabular}

upstream transcript and cloned in pAC245 at XhoI-KpnI sites. This construct was then transformed after a digestion by KpnI-SacI in ACY315 (orf19.2646 $\Delta / \Delta$ ) and SC5315 yielding to ACY402 and ACY401, respectively, in which the deleted allele of orf19.2646 was replaced by the upstream transcript cassette by double cross-over. For the construction of pAP4, a PCR was performed using primers 2646-postRACE3'Forward and 2646-postRACE3' Reverse (Table 3) on the genomic DNA of ACY294 to amplify the region corresponding to the downstream transcript and cloned in pAC245 at SacI-SacII sites. This construct was then transformed after a digestion by KpnISacI in ACY297 (orf19.2646 $\triangle$ /ORF19.2646) and SC5315 yielding ACY404 and ACY403, respectively. For pAC323 used to delete the region encoding the Tn7 upstream transcript, a PCR was performed on the $3^{\prime}$-end of the upstream transcript using primers 2646Del5'_R-bis-SacI and 2646Del5'_F-bis-SacII (Table 3) on BCY152 genomic DNA and cloned in pAC245 at SacI-SacII sites. For pAC321 used to delete the region encoding the Tn7 downstream transcript, a PCR was performed on the $5^{\prime}$ end of the downstream transcript using primers Deletion- $3^{\prime}$ F-KpnI and Deletion-3'-R-Xho (Table 3) on BCY152 genomic DNA of and cloned in pAC245 at KpnI and XhoI sites. All these plasmids were digested by KpnI and SacI before transformation in yeast strains. To delete the second allele of the Tn7 upstream or downstream regions, the SAT1 cassette was regenerated by the use of FLP recombinase, which is controlled through a maltose-inducible promoter (Reuss et al., 2004). 
TABLE 2 | List of plasmids.

\begin{tabular}{lll}
\hline Plasmid & Characteristics & References \\
\hline pAC245 & orf19.2646 deletion cassette & $\begin{array}{l}\text { Amorim-Vaz et al., } \\
2015\end{array}$ \\
& & Amorim-Vaz et al., \\
pAC253 & orf19.2646 WT revertant cassette & 2015 \\
& & This study \\
pAC339 & orf19.2646 885 bp revertant cassette & This study \\
pAP3 & pAC245/Tn7 upstream CDS & This study \\
pAP4 & pAC245/Tn7 downstream CDS & This study \\
pAC321 & pAC245/5' part of the downstream transcript & This study \\
pAC323 & pAC245/3' part of the upstream transcript & \\
\end{tabular}

\section{RNA Extraction RT-qPCR Analysis}

C. albicans strains were grown overnight in $3 \mathrm{ml}$ YEPD at $30^{\circ} \mathrm{C}$ under agitation. Fifty microliters of cultures were diluted in $5 \mathrm{ml}$ YEPD and incubated at $30^{\circ} \mathrm{C}$ until reaching $1.5 \times$ $10^{7}$ cells $/ \mathrm{ml}$. The cultures were then centrifuged $5 \mathrm{~min}$ at $13,000 \mathrm{rpm}$. The pellet was resuspended in $350 \mu \mathrm{l}$ RNA buffer

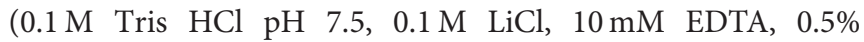
SDS) and transferred into tubes with screwed caps containing $200 \mu \mathrm{l}$ diethylpyrocarbonate (DEPC)-treated beads and $300 \mu \mathrm{l}$ phenol/chloroform/isoamyl alcohol (25:24:1). The tubes were shaken using the bead beater FastPrep-24 (MP biomedicals, Santa Ana, CA) with the following settings: speed $5 \mathrm{~m} / \mathrm{s}$ and time $5 \mathrm{~s}$. The tubes were next centrifuged for $1 \mathrm{~min}$ at full speed. The supernatant was transferred into tubes containing 250 $\mu l$ phenol/chloroform/isoamyl alcool (25:24:1) and tubes were vortexed for $10 \mathrm{~s}$ and centrifuged for $1 \mathrm{~min}$ at full speed. The supernatant was transferred into tubes containing $600 \mu \mathrm{l}$ ethanol (EtOH) $100 \%$ and kept at $-20^{\circ} \mathrm{C}$ for $30 \mathrm{~min}$ (or alternatives: $10 \mathrm{~min}$ in dry ice). The tubes were centrifuged for $2 \mathrm{~min}$ at $4^{\circ} \mathrm{C}$ and full speed. The RNA pellet was washed with $600 \mu \mathrm{l} \mathrm{EtOH}$ $70 \%$. The pellets were air-dried and resuspended in $50 \mu \mathrm{IDEPC}-$ water. An RNase inhibitor (RNasin ${ }^{\circledR}$, Promega, Switzerland) was added to the samples and they were stored at $-80^{\circ} \mathrm{C}$.

After RNA extraction and DNase treatment, the integrity of the RNA was verified using a BioAnalyzer (Agilent Technologies, Waldbronn, Germany).

RNA was first reverse transcribed into cDNA using the Transcriptor High Fidelity cDNA synthesis kit (Roche Applied Science, Penzberg, Germany). Briefly, 10 ng total RNA was added to Oligo dT $(60 \mu \mathrm{M})$ in a total volume of $11.4 \mu \mathrm{l}$. The mix was incubated for $10 \mathrm{~min}$ at $65^{\circ} \mathrm{C}$ and the tubes were stored back on ice. Reaction buffer (1X), deoxynucleotide mix (2 $\mu \mathrm{M}), 1,4$ Dithiothreitol (DTT; $10 \mu \mathrm{M}$ ), protector RNase inhibitor (20U), and transcriptor high fidelity reverse (1U) were added to the mix, incubated for $30 \mathrm{~min}$ at $50^{\circ} \mathrm{C}$ and inactivated for $5 \mathrm{~min}$ at $85^{\circ} \mathrm{C}$. The $\mathrm{qPCR}$ was performed using 100 -fold diluted $\mathrm{cDNA}$, Taq supermix with ROX (1X) (BioRad, Hercules, CA), primers, and probe at $100 \mathrm{nM}$ final concentration each. The two probes used, e.g., upstream (up-) and downstream (do-) (Table 3), were Taqman probes (FAM-TAMRA). StepOnePlus software was applied to analyse data (Applied Bioscience, Foster city, CA). Relative mRNA levels were calculated as previously described (Sierro et al., 2001) using the ACT1 housekeeping gene (Table 3).
TABLE 3 | List of primers.

\begin{tabular}{|c|c|}
\hline Name & Sequence $\left(5^{\prime}-3^{\prime}\right)$ \\
\hline 2646-start-Hind & GCGCAAAAGCTTATGGATAAGACAAATAGTCCAGGC \\
\hline 2646-insert1-xho & CGCGAACTCGAGAATTGATAATGGTTGGTGCTG \\
\hline 2646-insert2-xho & CGCGAACTCGAGAATACTCATGCCAGATATTGATG \\
\hline 2646-insert3-xho & CGCGAACTCGAGAATTGGAGCTGTTTGATCAGATAAC \\
\hline 2646-stop-sph & GCGCAAGCATGCTTATAGAAGATCATTGAAATCACC \\
\hline 2646-Sybr-RevB & GCACTCATGGCATCGGTGGCT \\
\hline 2646-promF-KPN & GCGCAAGGTACCGATATAACAATTATTTGTACACC \\
\hline $2646-+500$ & CCGCCACCACCACCG \\
\hline 2646-up-F & TCGGTCTAGTGCATCTCCTCA \\
\hline 2646-up-R & CTGCTGGAAATGGTGGTTGTG \\
\hline 2646-up-probe & ACCAACCACCGCCAATACAT \\
\hline 2646-do-F & CCACTCGTTACACCACATTTG \\
\hline 2646-do-R & TTCACCCGTGGGATCTTC \\
\hline 2646-do-probe & CCGATGCCATGAGTGCATTG \\
\hline Act1-F & ATAACGGTTCTGGTATGT \\
\hline Act1-R & CCTTGATGTCTTGGTCTA \\
\hline Act1-probe & CGGTGACGACGCTCCAAG \\
\hline 2646-P1-RACE & AACCACAACCACCATTCCAGCAGCAGCA \\
\hline 2646-P1b-RACE & AGGCACAAATCTCCATTCCAACGCCACCA \\
\hline 2646-P2b-RACE & TTAGTAGCCACCGATGCCATGAGTGCA \\
\hline 2646-P3-RACE & AACGTGCGGGTATTCAACATCACACCCA \\
\hline 2646-P3b-RACE & AAGATGCACTGCTGTTGCCGTTACCGTTG \\
\hline 2646-P4-RACE & TGGTGGCGTTGGAATGGAGATTTGTGCCT \\
\hline 2646-P4b-RACE & TGCTGCTGCTGGAAATGGTGGTTGTGGTT \\
\hline $\begin{array}{l}\text { GeneRacer oligo } \\
\text { dT primer }\end{array}$ & GCTGTCAACGATACGCTACGTAACGGCATGACAGTG(T) 24 \\
\hline GeneRacer $5^{\prime}$ & CGACTGGAGCACGAGGACACTGA \\
\hline
\end{tabular}

GeneRacer 5' nested primer

GeneRacer 3' primer

GeneRacer 3' nested primer

2646postRACE5'reverse

2646postRACE3'forward

2646-

\section{GGACACTGACATGGACTGAAGGAGTA}

GCTGTCAACGATACGCTACGTAACG

CGCTACGTAACGGCATGACAGTG

CGCAAACTCGAGCCGTTTATACCATCCAAATC

CGCAAACCGCGGCACGCATCTTCCCGACAACG

postRACE3'reverse

orf19.2646-up5

orf19.2646-down3

Primer N

Primer S

UAU-amplif-R

UAU-seq-R

2646-seq-5

2646Del5'_R-bis-

Sacl

2646del5'-F-bis-

Sacll

Deletion-3'-F-Kpnl

Deletion-3'-R- Xho
CGCAAAGAGCTCGCAGGTITACAAACCACATC

TCAATCAAGCCTCCTGTACCACCAC

CTCATTATTAGGAGTTGCTAACCA

ACTTATTGTCATAGTTAAGATCTATITG

TATTAGGAATIITGAGGTAAAGGTGGGGA

CTGTGCTACTGGTGAGG

GTCTTAGTGTTGACTGTC

ATGGAGTGTTGTCACC

CGCGAGCTCTATAGCCTCCATTAGATC

CGCCCGCGGCTAATTTCAATCCTAGCAC

AAACGCGGTACCAGAATTCTAATCCAACGG

AAACGCCTCGAGGATCTGATTGATATTAAACTC 


\section{RNA Ligase-Mediated-Rapid Amplification of CDNA Ends (RLM-RACE)}

The RLM-RACE was performed according to manufacturer's protocol (GeneRacer ${ }^{\mathrm{TM}}$ Kit, Thermofisher, Switzerland). Briefly, total RNA $(5 \mu \mathrm{g})$ from ACY293 and ACY294 were used to perform the RACE analysis. HeLa total RNA (1 $\mu \mathrm{g})$ was used as internal control of the kit. The three samples were first treated with calf intestinal phosphatase (CIP) in order to dephosphorylate RNAs that are either truncated mRNA with a polyA-tail but not $5^{\prime}$ capped or other non-mRNA. The dephosphorylated RNA was then precipitated before being treated with tobacco acid pyrophosphatase (TAP) to remove the $5^{\prime}$ cap structure of the mRNA, thereby exposing the $5^{\prime}$ phosphate. The RNA was next ligated with GeneRacer RNA Oligo to the $5^{\prime}$ phosphate using T4 RNA ligase. The RNA was reverse transcribed using a GeneRacer Oligo dT Primer. This resulted in the creation of a RACE-ready first-strand cDNA. GeneRacer $5^{\prime}$ primers and reverse gene specific primers (Reverse GSP) were used to amplify the $5^{\prime}$ ends, while GeneRacer $3^{\prime}$ primers and forward gene specific primers (Forward GSP) were used to amplify the $3^{\prime}$-ends. The PCR was performed using the hot start Platinum Taq DNA Polymerase High Fidelity. The annealing temperature was $60^{\circ} \mathrm{C}$ and the elongation time varied depending on the expected product's length. If needed, GeneRacer nested primers that are located closer to the gene of interest were provided. Other GSP closer to the $5^{\prime}$ - or $3^{\prime}$-ends were used to obtain a PCR product in combination with the GeneRacer primers. The PCR products were purified using the Nucleospin Gel and PCR Clean-up (Macherey-Nagel, Switzerland) and were ready for cloning.

PCR products were cloned into $\mathrm{pCR}^{\mathrm{TM}} 4-\mathrm{TOPO}^{\circledR}$ TA vectors (Thermo Fisher Scientific, Switzerland) following instruction of the manufacturer. For the upstream transcript, GR5nestedP4b, GR3-P1, and GR3-P1b yielded Topo6-28, Topo2-6, and Topo3-11 plasmids respectively. For the downstream transcript, GR5nested-P3b, GR3-P2b yielded Topo8-36, -39, -40, and Topo4-16, -17, -19 , respectively.

\section{Sequencing}

Sequencing of plasmids was performed using an ABI Prism $3130 \mathrm{XL}$ automated DNA sequencer (Perkin-Elmer/Applied Biosystems, Foster City, CA) with a BigDye terminator cycle sequencing kit (version 1.1, Applied Biosystems) according to the manufacturer's protocol using primers from the GeneRacer ${ }^{\mathrm{TM}} \mathrm{Kit}$ (M13F, M13R, T3, and T7) diluted 20-fold (5 $\mu \mathrm{M})$.

\section{Mice Infection and Ethic Statement}

All animal experiments were performed at the University Hospital Center of Lausanne with approval through the Institutional Animal Use Committee, Affaires Vétérinaires $\mathrm{du}$ Canton de Vaud, Switzerland (authorization $\mathrm{n}^{\circ}$ 1734.3), according to decree 18 of the federal law on animal protection. For all mice experiments, female BALB/c mice (6 weeks old; Charles River France) were housed in ventilated cages with free access to food and water. The strains of $C$. albicans were grown in individual tubes for $16 \mathrm{~h}$ under agitation at $30^{\circ} \mathrm{C}$ in YEPD medium. Each strain was subsequently diluted 100-fold in YEPD medium and grown overnight under agitation at $30^{\circ} \mathrm{C}$. Overnight cultures were washed twice with PBS (137 mM NaCl, $2.7 \mathrm{mM}$ $\mathrm{KCl}, 10 \mathrm{mM} \mathrm{Na} 2 \mathrm{HPO} 4,1.8 \mathrm{mM} \mathrm{KH} 2 \mathrm{PO} 4)$ and resuspended in $5 \mathrm{ml}$ PBS. The concentration of each culture was measured through optical density, and each strain was diluted to $8 \times 10^{5}$ cells $/ \mathrm{ml}$. The mice were injected through the lateral tail vein with $250 \mu \mathrm{l}$ of cell suspension. At 3 days post-infection (dpi), the kidneys were recovered, and the Colony-forming units (CFU) were determined as previously described (Vandeputte et al., 2011). The strains were tested in groups of 5 mice and repeated twice. Experiment results were pooled and thus expressed as percent of CFU obtained with the isogenic wild-type strain used in each experiment. The limit of detection was $50 \mathrm{CFU}$ per 2 kidneys.

\section{Galleria Mellonella Infection}

Galleria mellonella fungal burden were assessed as described previously (Amorim-Vaz et al., 2015). Briefly, the different strains were grown overnight in $5 \mathrm{ml}$ YEPD. The cultures were next washed in PBS and re-suspended in PBS complemented with $200 \mu \mathrm{g} / \mathrm{ml}$ ampicillin, at $5 \times 10^{6}$ cells/ml. Each larva (350$400 \mathrm{mg}$ ) was injected through the last left proleg with $40 \mu \mathrm{l}$ of inoculums $\left(2 \times 10^{5}\right.$ cells $)$. After $24 \mathrm{~h}$ in the dark at $30^{\circ} \mathrm{C}$, each larva was introduced into screw-cap tubes containing a metal bead (stainless steel, $7 \mathrm{~mm}$, [VWR International]). The tube was closed and firmly agitated performing three $10 \mathrm{~s}$ cycles at $6.5 \mathrm{~m} / \mathrm{s}$ with a MP FastPrep ${ }^{\circledR}-24$ equipment (MP Biomedicals). The homogenate was resuspended in $5 \mathrm{ml}$ PBS in a $14 \mathrm{ml}$ Falcon tube, serially 10 -fold diluted in PBS. One hundred microliters were plated on YEPD-chloramphenicol $(50 \mu \mathrm{g} / \mathrm{ml})$ plates and incubated at $30^{\circ} \mathrm{C}$ for $24-48 \mathrm{~h}$. CFU were then enumerated on three plates and means were calculated. Fungal loads were expressed as CFU per larvae. The limit of detection was $50 \mathrm{CFU}$ per larvae. For feasibility of the experiments, all the mutants of one analysis could not be performed at the same time due to the high number of individual to manage. Several experiments were performed with different mutants always including the isogenic wild-type strains as a positive control. Some level of variability was observed from one experiment to another even with the same mutants, therefore data of fungal burden are always expressed as a percentage of the isogenic wild-type strain present in each individual experiment.

\section{RESULTS}

\section{Analysis of the Orf19.2646 Tn7 Interrupted Mutant of the Collection}

In the TIGR database (Chamilos et al., 2009), 3 different mutants for orf19.2646 were identified. Three possible insertions were described: ins CAGCU92 (position +460), ins CAGGF84 (position +885), and ins CAGE210 (position +1151) (Figure 1A). To determine which mutant was corresponding to strain BCY152 of the TF mutant collection (Vandeputte et al., 2011; Amorim-Vaz et al., 2015), a discriminative PCR was performed using a forward primer (orf19.2646-start-HindIII) with different reversed primers (2646-insert1, 2646-insert2, 2646-insert3, 2646-Sybr-RevB, and 2646-stop-Sph) (Figure 1A). 

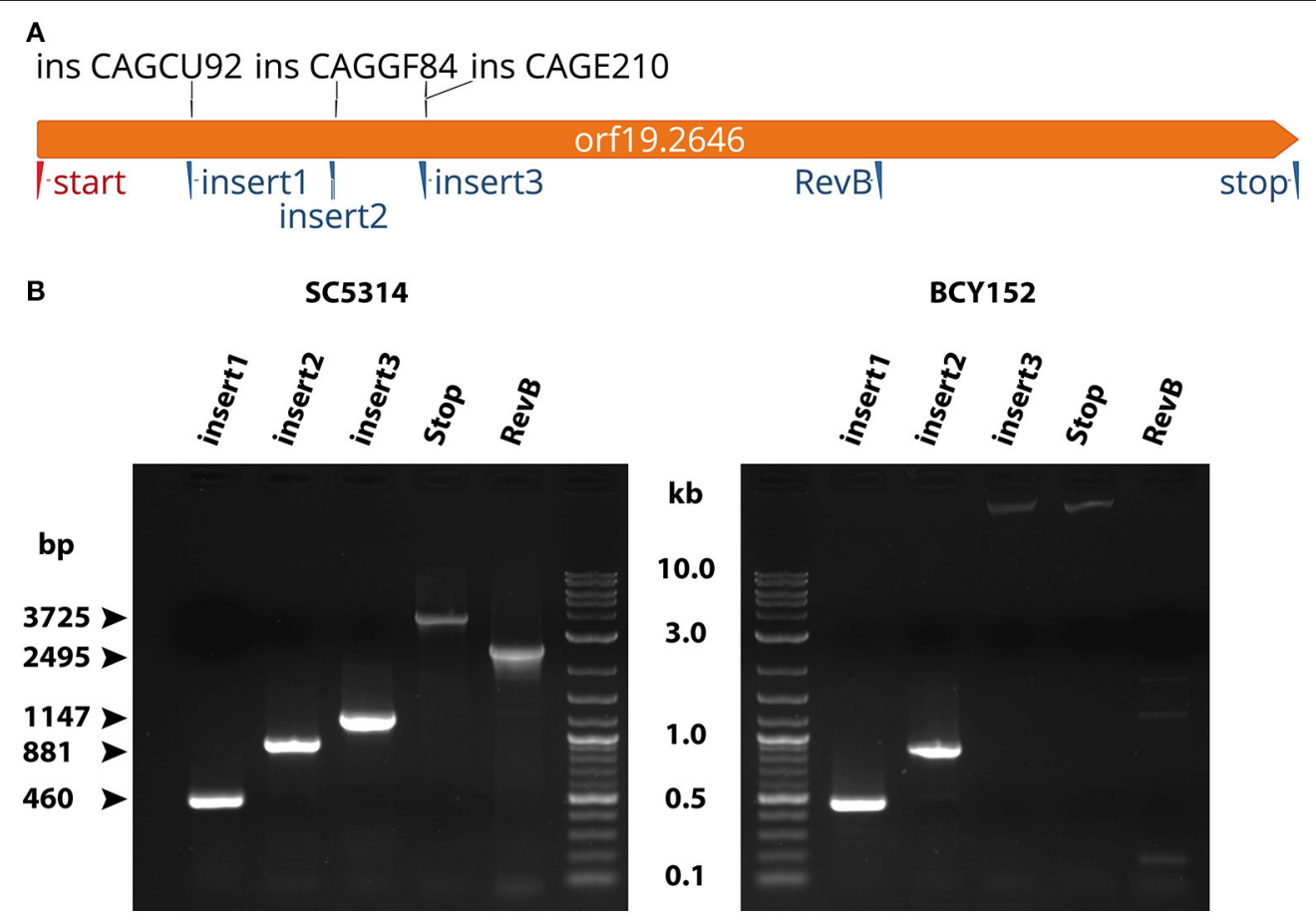

FIGURE 1 | Analysis of the Tn7-UAU cassette location in orf19.2646. (A) Map of orf19.2646 gene with the three different possible insertions (ins CAGCU92, ins CAGGF84, and ins CAGE210) of the Tn7-UAU cassette as sequenced by TIGR. Triangles represent position of the forward (red) and reverse (blue) primers used to detect the insertion of the Tn7-UAU cassette. (B) PCR amplification on SC5314 DNA (left panel) and orf192646 Tn7 mutant (BCY152) DNA (right panel). Each PCR was performed using the forward primer "start" and reverse primers as indicated.

As a positive control DNA from strain SC5314 was used. PCR products obtained using the reverse primers insert1 and insert2 yielded products of 460- and 881-bp for both DNA, respectively (Figure 1B). In contrast, PCR with insert3, RevB, and Stop primers yielded products of 1147-, 2495-, and 3725-bp for SC5314 DNA only (Figure 1B), respectively. For BCY152 amplification products were observed above $10 \mathrm{~kb}$ with reverse primers insert 3 and RevB but no signals were observed in the presence of Stop primer (Figure 1B). These results indicate that the Tn7 cassette present in the BCY152 orf19.2646 mutant was positioned between the insert 2 and insert 3 primers, thus corresponding to the CAGGF84 TIGR clones in which the Tn7 cassette was predicted to be inserted at position +885 relative to the ATG. Thus, the first 885-bp of orf19.2646 are still present in this mutant and could lead to the production of a chimeric transcript. This truncated transcript might be responsible for the low fungal burden phenotype observed in previous studies (Vandeputte et al., 2011; Amorim-Vaz et al., 2015).

\section{Analysis of the orf19.2646 Deletion Mutant Carrying a Truncated Transcript Corresponding to the First 295aa}

To test the above-mentioned hypothesis, we amplified the first 885-bp (corresponding to 295 aa of orf19.2646 in addition to 1,000-bp upstream of orf19.2646 ATG (using primers 2646promF-KPN and 2646-insert2-xho) and cloned it into a cassette to allow recombination with orf19.2646 in the deletion mutant. We called this truncated version of orf19.2646 the "S" (short) allele. The S construct was integrated at the orf19.2646 locus by homologous recombination in the deletion mutant. This strain was designated as "S-revertant" (Figure 2). In addition, a wildtype (WT) copy of orf19.2646 was introduced by transformation of the pAC253 revertant cassette (Amorim-Vaz et al., 2015). This last strain was designated as "S + WT-revertant" (Figure 2).

As a first analysis, the expression of the construct was verified by RT-qPCR using primers and probes hybridizing upstream (up) and downstream (do) the insertion of the Tn7 cassette (up-F, up-R and up-probe, and do-F, do-R and do-probe, respectively) (Figure 2A). We tested the transcription of DNA upstream and downstream the first 885-bp in SC5314, the deletion mutant, the WT-revertant, and finally in the two new strains obtained in this study (S- and S + WT-revertants). We could clearly observe that the upstream probe gave a signal for all strains except the deletion mutant (Figure 2B), indicating that a transcript is produced in the S-revertant. In contrast and as expected, the Tn7 downstream probe gave a signal only for strains carrying a orf19.2646 wildtype copy (SC5314, WT-, and S + WT-revertants; Figure 2B). We could also observe that the WT-revertant displayed a decreased orf19.2646 expression as compared to SC5314. This can be explained by a gene dosage effect, since only one allele was re-introduced into the revertant strain.

Next, the fungal burden phenotypes of all the five above described strains were tested in mice (Figure 3). The S-revertant, 
A
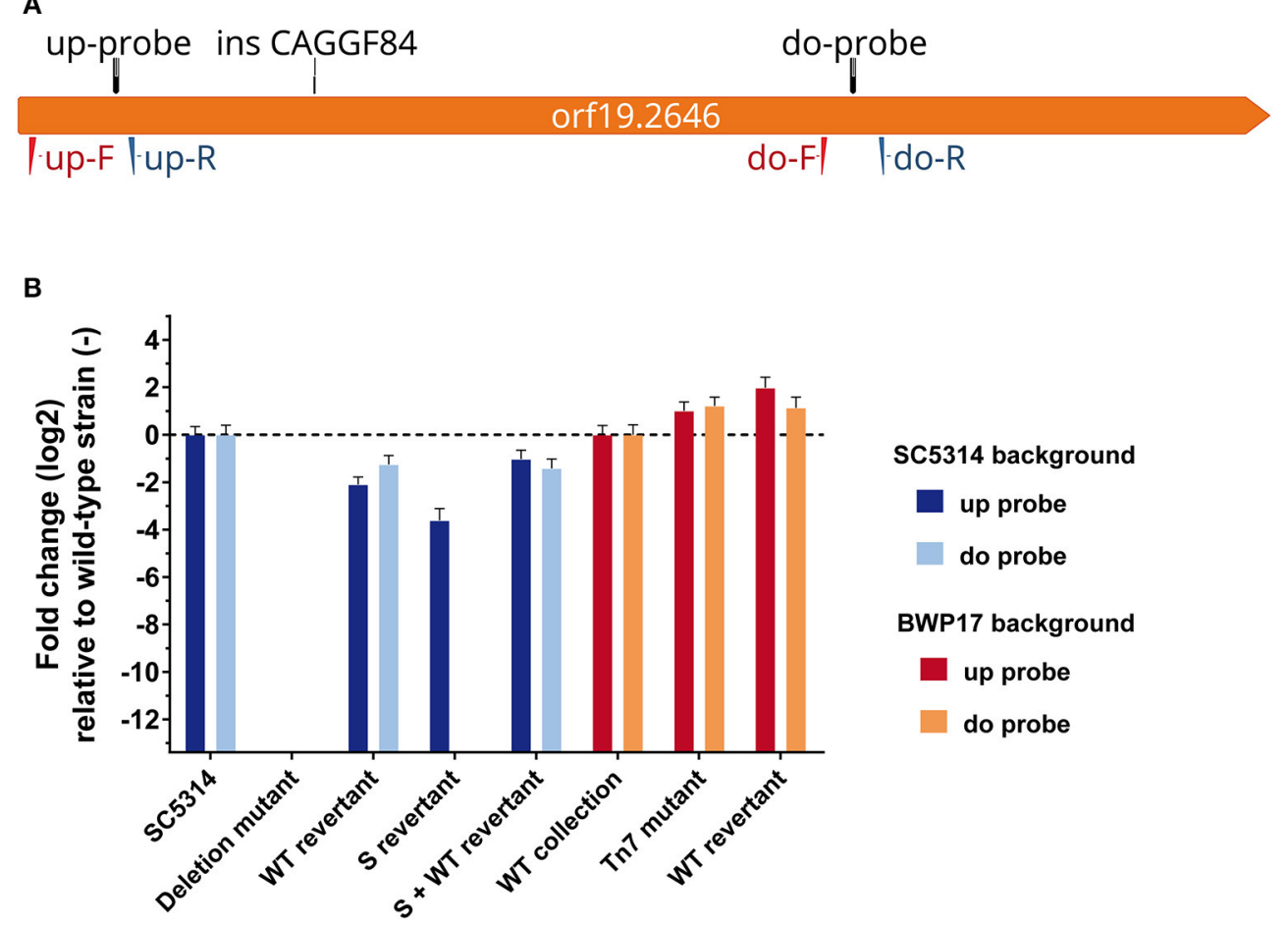

FIGURE 2 | mRNA level of Tn7 putative upstream and downstream transcript. (A) Map of orf19.2646 with the position of the upstream (up-) and downstream (do-) primers and up- and do-TaqMan ${ }^{\mathrm{TM}}$ probes. (B) RT-qPCR relative quantification of upstream and downstream transcripts. Results are expressed relative to the quantity measured for the isogenic wild-type strains. Each bar-plot corresponds to the mean of two biological samples measured in triplicate.

in contrast to SC5314, the deletion mutant and the WT-revertant, displayed lower fungal burden (Figure 3). This phenotype was reverted in this strain by an additional wild-type copy of orf19.2646 (Figure 3, S+WT-revertant). This result is consistent with the hypothesis that an unexpected transcript corresponding to a truncated protein due to the Tn7 insertion might be responsible for the observed low fungal burden phenotype in the orf19.2646 mutant of the collection.

\section{mRNA Expression Levels in the Different Strains}

To further verify this hypothesis, the production of a transcript upstream of the $\operatorname{Tn} 7$ in the orf19.2646 Tn7-UAU mutant was analyzed by RT-qPCR (Figure 2). The revertant strain and the isogenic wild-type strain were analyzed as controls (Figure 2B, BWP17 background). As expected in the wildtype and revertants strains, amplification at both the upstream and downstream Tn7 flanking regions could be observed. Amplification occurred at the upstream Tn7 flanking regions in the Tn7 mutant strain. Unexpectedly, we also observe an amplification product at the downstream region of the Tn7 mutant (Figure 2B). In the Tn7 mutant, the amount detected of both transcripts appeared to be higher than in the isogenic wild-type strain. This strongly suggests the presence of unexpected transcripts in the orf19.2646 Tn7mutant not only upstream the Tn7-UAU cassette insertion and corresponding to a truncated protein but also downstream of this cassette.

\section{Identification of Tn7 up-, and Downstream Transcript Sequences}

To further investigate the sequences of these two transcripts and to analyse whether they correspond to mRNA with all the features enabling the production of a protein, the Rapid amplification of cDNA ends (RACE) technique was used. This technique provides the sequence of an RNA transcript from a small known sequence within the transcript to the $5^{\prime}-\left(5^{\prime}\right.$ RACE-PCR) or $3^{\prime}$-end $\left(3^{\prime}\right.$ RACE-PCR) of the RNA. Performing both types of analysis provides the entire sequence of the putative Tn7 upstream and downstream mRNA.

As shown in Figure 4A, the GSP forward primers (P1, P1b, and P2b) were used with the GeneRacer $3^{\prime}$ (GR3) primer for the $3^{\prime}$ end cloning of the transcripts, while the GSP reverse primers (P3, P3b, P4, and P4b) were used with the GeneRacer 5' (GR5) primer for the $5^{\prime}$ end cloning of the transcripts. The strains used to perform the RACE analysis were the wild-type strain of the collection (ACY293) as a positive control and the Tn7 mutant (ACY294).

First, and to validate the procedure, the GeneRacer was carried out on RNA from HeLa cells with GSP allowing the amplification of the $\beta$-actin transcript. The expected sizes for both combinations of primers were obtained at $0.9 \mathrm{~kb}$ 


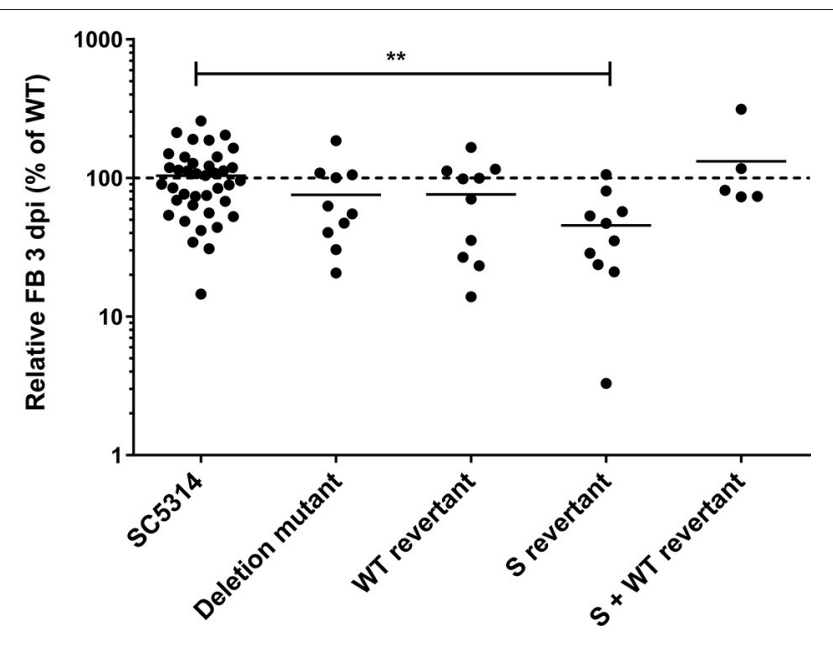

FIGURE 3 | Role of the 885 bp-truncated orf19.2646 on mouse kidney fungal burden (FB). For each individual mouse, the FB in the kidneys is expressed as a percentage relative to the mean of the wild-type group in the same experiment. All experiments are pooled in this graph. The mean value of each group is indicated with a black bar. The dotted line indicates the level of the mean of the wild-type strain set at $100 \%$. Each mutant was tested twice using five mice each time (except for S+WT revertant and SC5314 wild-type strains). Statistical analyses were performed using a Mann-Whitney test to assess CFU differences relative to SC5314 (GraphPad Prism 7.02). The stars indicate the level of statistical significance: ${ }^{* \star} p<0.01$. S stands for orf19.2646 "short" version of $885 \mathrm{bp}$.

for the $5^{\prime}$-end and $1.8 \mathrm{~kb}$ for the $3^{\prime}$-end (Figure $4 \mathrm{~B}$, left panel), thus indicating that the RACE procedure was performed correctly. Second, to verify whether the whole mRNA was reverse transcribed correctly, a PCR was performed using the GSP primers $\mathrm{P} 1$ and $\mathrm{P} 4$. As expected these two primers were able to obtain a PCR product of $294 \mathrm{bp}$ on the Tn7 upstream region (Figure 4B, middle panel), thus indicating that the reversetranscription step was also correctly performed on the Tn7 mutant C. albicans RNA.

GR-GSP PCR was then performed to amplify the end of the putative Tn7 upstream and downstream transcript (Figure 4A). With regards to the amplification of the $3^{\prime}$-end of the mRNA, an amplification for both the upstream (GR3-P1 and GR3-P1b), and the downstream (GR3-P2b) transcripts was obtained. In the case of the upstream transcript, products of 1,000 bp for P1GR3, and 800 bp for P1b-GR3 were observed (Figure 4B, middle panel). For the downstream transcript, a product of 1,000 bp was observed for P2b-GR3 (Figure 4B, middle panel). Regarding the $5^{\prime}$-end of this transcript, no amplification products were obtained for the upstream and downstream transcripts. We thus performed nested PCR on the product of P4-GR5 and P3-GR5 PCR products using internal primers $\mathrm{P} 4 \mathrm{~b}$ and $\mathrm{P} 3 \mathrm{~b}$, respectively, with the GR5-nested primer provided in the RACE kit. Nested PCR yielded a product of $500 \mathrm{bp}$ for the upstream transcript (P4b-GR5nested, Figure 4B, right panel) and of 700 bp for the downstream transcript (P3b-GR5nested, Figure 4B, right panel). Thus, both $5^{\prime}$ - and $3^{\prime}$-ends of both transcripts were amplified. To determine the sequences of the transcript ends, the five PCR products were TOPO cloned and sequenced.
The upstream transcripthas all the features of an mRNA (for complete sequences, see File S1): a $5^{\prime}$-untranslated region (UTR), a coding sequence (CDS), a $3^{\prime}$-UTR and a poly-A tail. Note that the cap could not be detected since it was removed during the RACE procedure. Then, the sequences were aligned together and with the orf19.2646 Tn7 interrupted sequence as deduced from the TIGR description (Figure 5A). The two sequences did not perfectly align with the predicted orf19.2646 Tn7 interrupted sequence. A gap of $180 \mathrm{bp}$ was observed downstream of the TIGR described Tn7 insertion. It appeared that the Tn7 cassette was 180 bp downstream at position +1065 bp from the start of orf19.2646. After redesign of the map at the orf19.2646 Tn7 interrupted locus, the upstream transcript could be aligned with this new sequence (Figure 5B). The start of the CDS is identical to orf19.2646. The stop codon is located in the Tn7R sequence. The $5^{\prime}$-UTR is part of the orf19.2646 promoter and the $3^{\prime}$-UTR is situated at start of the UAU cassette.

The downstream transcript possessed also all features of an mRNA with $5^{\prime}$ - and $3^{\prime}$-UTR, CDS, and PolyA sequences (see File S1). Then this downstream transcript was aligned with the orf19.2646 Tn7 interrupted sequence (Figure 5). It aligned within the orf19.2646 CDS, the Tn7L sequence being probably part of the promoter of this transcript (Figure 5).

\section{Reconstruction of the Upstream and Downstream Transcript Mutants}

After the identification of unexpected transcripts, their role in the fungal burden phenotype of a $C$. albicans strain was addressed. For this purpose, two plasmids (pAP3 and pAP4) were designed from the orf19.2646 deletion cassette pAC245 (Figure 6A, for details see Material and Methods). The cassettes were inserted at the orf19.2646 locus by double cross-over in a deletion mutant (ACY315). As the $3^{\prime}$-UTR of the downstream transcript was no longer present in the deletion mutant, this cassette was transformed into the orf19.2646 heterozygous mutant (ACY297) to replace the remaining wild-type allele. Two strains: one carrying only the upstream transcript allele (ACY402; uprevertant), and another with the downstream transcript allele (ACY404; do-revertant) were thus obtained. A mutant with two transcripts was also constructed (ACY405; up+do-revertant). One allele would possess the upstream transcript allele, while the other would possess the downstream transcript allele. As a control, both constructs were transformed into SC5314 (data not shown). All strains were verified by southern-blot analysis (data not shown).

To verify that the new mutant strains ACY402, ACY404, and ACY405 expressed the upstream and downstream transcripts, we performed the same RT-qPCR as above (Figure 6B). The strain SC5314, the wild-type collection strain (WT collection), the S-revertant and the Tn7 mutants were used as controls. As expected, those strains carrying at least one wild-type allele of orf19.2646 (SC5314 and WT collection), as well as the Tn7 mutant, displayed amplification of both the upstream and downstream regions. As previously observed, the S-revertant displayed amplification only for the upstream region. For the up-revertant, we observed a signal for the upstream probe, but 


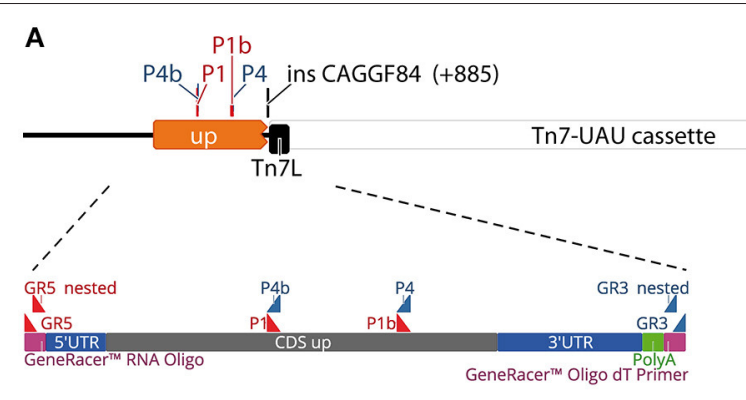

B

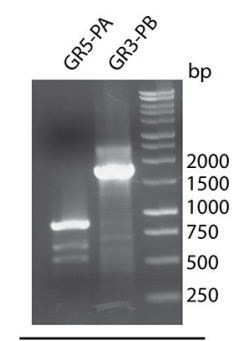

HeLa total RNA control
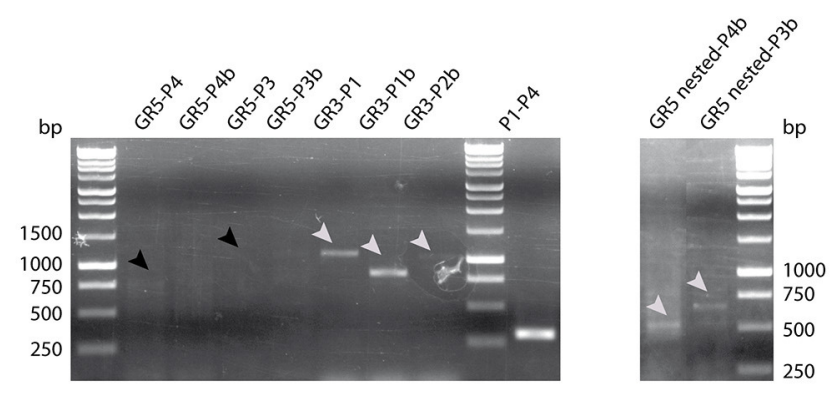

FIGURE 4 | RACE analysis. (A) Map of Tn7 mutated orf19.2646. Orf19.2646 (gray) is interrupted by the Tn7-UAU cassette (white bar). The putative transcripts drawn between the dotted lines have been ligated to a $5^{\prime}$ GeneRacer ${ }^{T M}$ Oligo and $3^{\prime}$ GeneRacer ${ }^{T M}$ Oligo dT primers (purple). The forward primers (P1, P1b, and P2b, red triangles) are designed to amplify a products with the GeneRacer $3^{\prime}$ primer (GR $3^{\prime}$ ), while the reverse primers (P3, P3b, P4, and P4b, blue triangles) are designed to amplify products with the GeneRacer $5^{\prime}$ primer (GR 5'). (B) Left panel: Control PCR with HeLa total RNA. Middle panel: RACE PCRs performed with the GR5', the GR3' primers and with the P1-P4 primers on SC5314 RNA as control of reverse transcription. Right panel: Nested PCR performed on the GR5-P4 and GR5-P3 PCR products (black arrows). Topo cloning was performed on the products indicated by a white arrow.

it was 20 times reduced as compared to SC5314. For the dorevertant, we observed a signal for the downstream probe, but 8 times lower compared to SC5314 (Figure 6B). The strain carrying both the upstream and the downstream transcript alleles (up+do-revertant) showed signals for both the upstream and the downstream-probe which were 8 - and 30 -fold lower than SC5314, respectively. These results are in contrast to the level of expression measured in the Tn7 mutant, for which both signals were at least 10-fold higher than wild-type. All together, these data indicated that the reconstructed upstream and downstream transcripts were successfully produced, although to lower amounts as compared to wild-type (Figure 6B).

It was next investigated whether these transcripts were responsible for the low fungal burden observed in the orf19.2646 Tn7 mutant, both in mice and in the mini-host model $G$. Mellonella (Amorim-Vaz et al., 2015). For this purpose, the ability of the different up- and do-revertants to invade larvae of G. Mellonella (Figure 6C) was tested. Regarding the Tn7 mutant, we observed a significantly reduced fungal burden as compared to wild-type as previously published (Amorim-Vaz et al., 2015). For both the up- and do-revertants, no significant change in fungal burden was observed as compared to wild-type strain. Nevertheless, when both the upstream and downstream transcripts were expressed together in the same strain (up+dorevertant), a significant reduction of fungal burden was measured
(ANOVA adjusted $p=0.037$; Mann-Whitney $p=0.0008$ ). Fungal burden observed with the strain expressing both transcripts was $60 \%$ reduced when compared to wild-type, while a $90 \%$ reduction in fungal burden was observed with the Tn7 mutant. These data suggest that the presence of both transcripts is required to recapitulate a low fungal burden phenotype, although not at the same magnitude as for the Tn7 mutant.

\section{Deletion of the Tn7 Upstream and Downstream Flanking Region in the orf19.2646 Tn7 Mutant}

Unexpected transcripts of orf19.2646 Tn7 mutant were deleted to determine the level of fungal burden phenotype in G. mellonella. Strains derived from the Tn7 mutant lacking either the Tn7 upstream, downstream or, both regions (ACY430, ACY422, and ACY428 respectively) were obtained. The genetic composition of the strains was verified by southern-blot (data not shown). G. mellonella were infected with the mutants and compared with larvae infected with the orf19.2646 Tn7 mutant and the isogenic wild-type strain (Figure 7). Low fungal burden of the Tn7 mutant as compared to wild-type (adjusted $p<0.0001$ ) was observed again in this model. The strains deleted for the $\operatorname{Tn} 7$ upstream or downstream regions also displayed a fungal burden higher than the Tn7 mutant (ANOVA adjusted $p=0.1$, and 

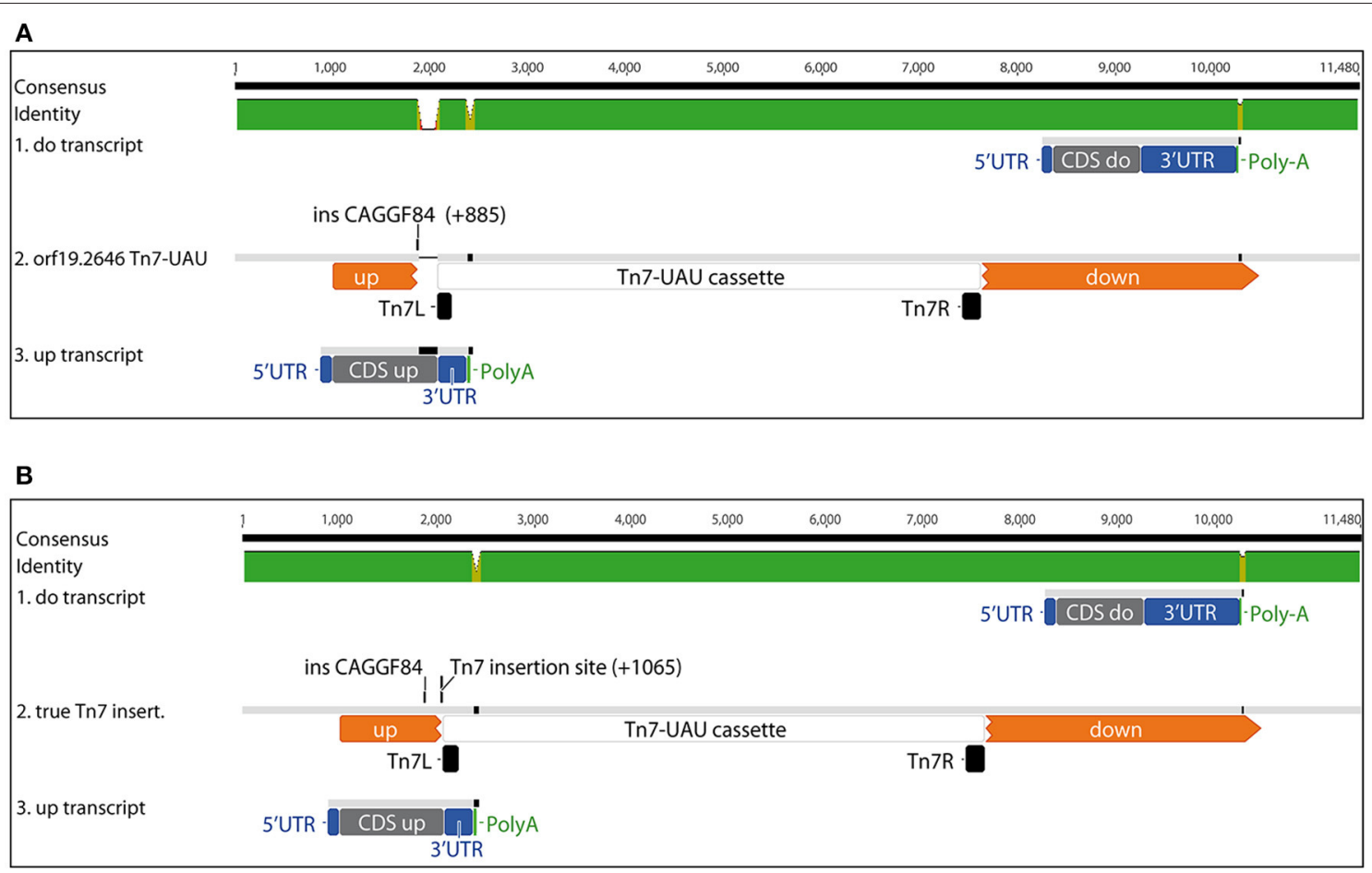

FIGURE 5 | Mapping of the upstream (up) and downstream (do) Tn7 transcripts. (A) Mapping of the reconstituted upstream and downstream transcript sequences on the CAGGF84 TIGR sequence, (B) Newly deduced sequence for the orf19.2646 Tn7-UAU insertion locus and alignment with the identified upstream and downstream transcripts.

$p=0.04$, respectively), even if only the strain deleted for the downstream region gave a significant result. The strain deleted for both the upstream and the downstream regions (ACY428) displayed a fungal burden similar to the isogenic wild-type which was significantly higher than for the Tn7 mutant (Figure 7; ANOVA adjusted $p=0.0047)$. Taken together, these results confirmed the role of these two unexpected transcripts upstream and downstream the Tn7-UAU cassette in the low fungal burden phenotype observed in mice and G. mellonella.

\section{DISCUSSION}

The aim of this study was to analyse the $\operatorname{Tn} 7$ interruption mutation of the orf19.2646 C. albicans mutant in order to determine the cause of its low fungal burden phenotype observed in vivo which was not recapitulated by a null mutant. One possible explanation for this phenotype was that a truncated protein was formed as a result of the Tn7-UAU cassette insertion. Indeed, as previously described for several Tn7 mutants, we could demonstrate that a Tn7 upstream transcript was formed corresponding to a truncated form of the orf19.2646 transcript. Surprisingly, we also found that downstream of the Tn7 cassette, another transcript was formed corresponding to a complete unexpected transcript. We determined the sequence of these two transcripts by RACE analysis. After reintroducing the transcripts into an orf19.2646 deletion mutant, we observed that both the upstream and downstream transcripts were responsible for the low fungal burden phenotype observed with the Tn7 mutant. Similar results were obtained when the corresponding sequences for the 2 transcripts in the Tn7 orf19.2646 mutant were deleted simultaneously. An exact determination of the insertion site of the Tn7-UAU cassette in this mutant was achieved which does not correspond to previous annotations by the TIGR consortium.

As previously described for other Tn7 mutants, the STOP codon of the unexpected upstream mRNA was found to be located within the Tn7 transposon. Indeed, Tn7 sequences (L or $\mathrm{R}$ ) have been previously shown to contain stop codons in all the 3 frames (Lo et al., 2003). We were able to identify a putative PolyA signal (AUAAA) in the $3^{\prime}$-UTR of the transcript 103 bp upstream of the polyA sequence. It has been previously described that in contrast to the mammalian PolyA signal located -35 to $-10 \mathrm{nt}$ upstream of the polyA sequence, the PolyA signal in yeast is more flexible in term of position and sequence (Moqtaderi et al., 2013). We were able to locate a putative TATA box with a non-canonical sequence 85 nucleotides upstream of the transcription site of this upstream transcript. However, initiation of transcription in yeast does not necessarily require a TATA box and no clear consensus sequence exists for an effective yeast TATA box (Basehoar et al., 2004).

Sequencing of the downstream transcript revealed that it was composed of part of the orf19.2646 sequence. To our knowledge, the occurrence of unexpected transcripts downstream of a Tn7 sequence has not been previously described. We hypothesized that the $\operatorname{Tn} 7$ sequence was part of the promoter region. This 

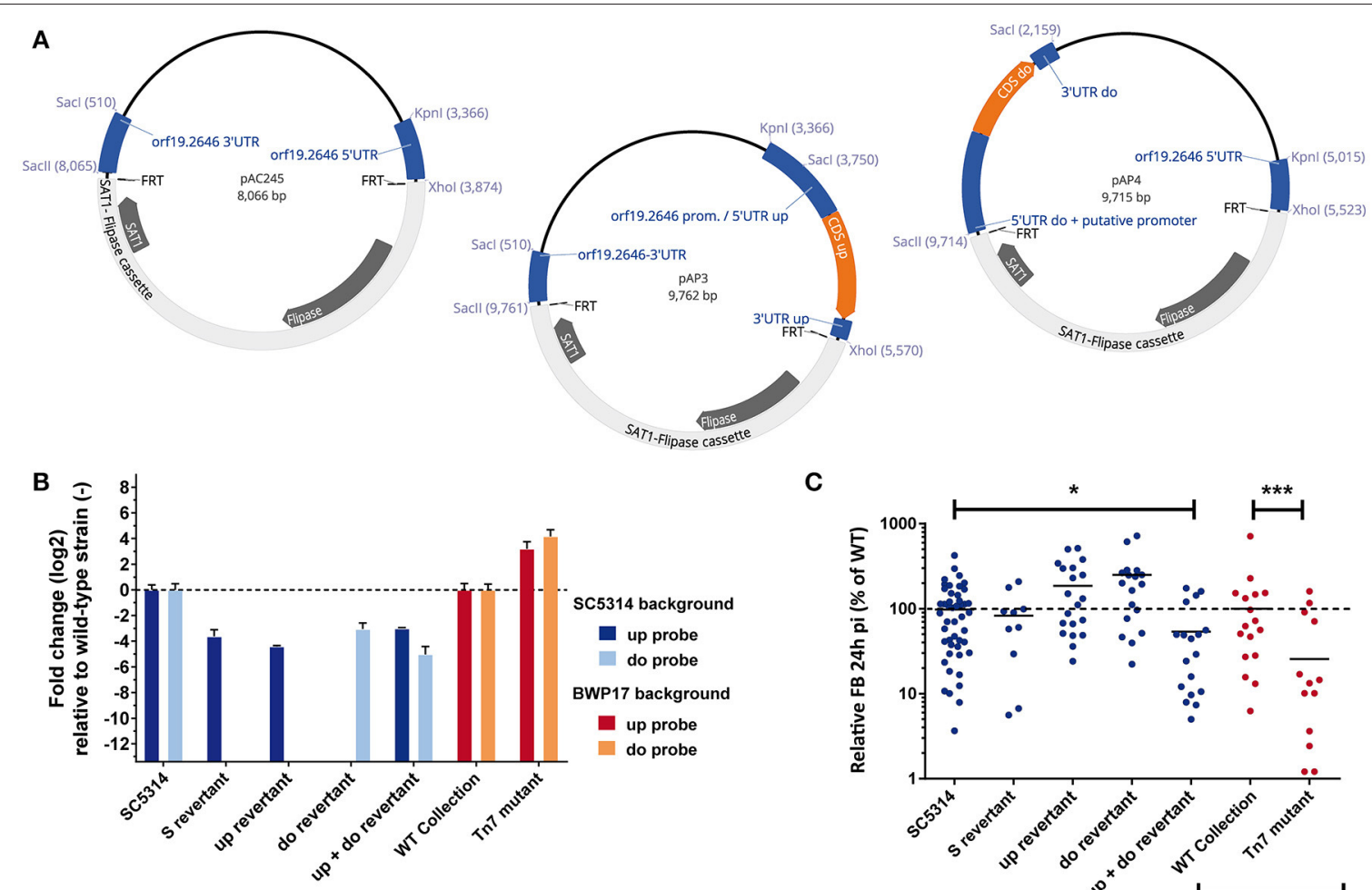

C

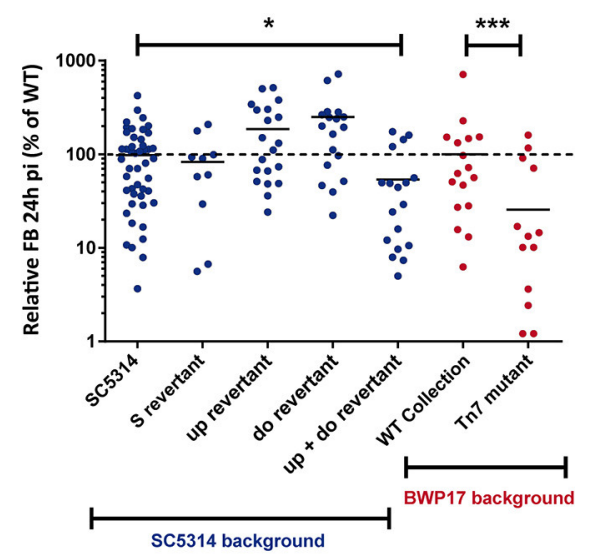

FIGURE 6 | Identification of the Tn7 up-, and downstream transcript sequences and role in the fungal burden phenotype. (A) Maps of the disruption cassettes used to introduce upstream and downstream transcripts in the deletion mutant. pAP3 and pAP4 were constructed from pAC245 by replacement of either orf19.2646 5'-UTR or $3^{\prime}$-UTR, respectively. (B) mRNA level of Tn7 putative upstream and downstream transcripts. Results are expressed relative to the quantity measured for the isogenic wild-type strain. Each bar-plot corresponds to the mean of two biological samples measured in triplicates. (C) G. mellonella fungal burden for each individual larva. The fungal burden is expressed as a percentage relative to the mean of the wild-type group in the same experiment. All experiments were pooled in this graph. The mean value of each group is indicated with a black bar. The dotted line indicates the level of the mean of the wild-type strain set at $100 \%$. Each mutant was tested at least twice using 10 larvae each time, except for the S-revertant which was tested only twice with 5 larvae (missing dots correspond to dead larvae). Statistical analyses were performed using a ROUT analysis to remove outliers (Motulsky and Brown, 2006), followed by a Mann-Whitney test to assess CFU differences relative to the wild-type strain (GraphPad Prism 7.02). The stars indicate the level of statistical significance: ${ }^{*} p<0.05,{ }^{* \star \star} p<0.001$.

was based on the observation that the coding sequence of the downstream transcript has a different reading frame than that of the wild-type gene, and that no gene locus has been documented in this region by the Candida Genome Database (CGD). The mRNA produced does not correspond to any known protein or protein domain. To verify the hypothesis that the Tn7 sequence was part of the promoter region, we could delete part of the transposon and test whether a transcript is still produced. In the meantime we could identify a putative PolyA signal (AUAAA) in this transcript situated 6 bases upstream of the PolyA sequence. As for the upstream transcript, we were unable to localize a canonical TATA box upstream of the transcription start site.

As previously described for other strains (Amorim-Vaz et al., 2015), the S-revertant strain did not appear to behave significantly differently from its isogenic wild-type strain after infection of G. mellonella, which is in contrast to the mouse experiments (Figures 3, 6C). Such results were attributed to the higher variability observed in G. mellonella than in mice, which might be due to the inbred lineages of mice in contrast to Galleria larvae. However, this result is in agreement with the up- and do-revertant results in G. mellonella. As for the S-revertant, the up-revertant was not sufficient to confer a significantly lower fungal burden in the $G$. mellonella larvae than for the wild type strain. The do-transcript effect is needed to obtain a phenotype sufficiently pronounced to be significantly different from the wild type strain in Galleria. However, the decrease of fungal burden observed with the Tn7 mutant was more pronounced than for the up + do-revertant (Figure 6C).

These results could be explained by the difference in expression of the up- and do-transcripts in both strains when compared to their respective wild-type strains (Figure 6B). The two strains do not have the same genetic background, one deriving from the BWP17 and the other from the SC5314 strain. Even though both parental strains are genetically related, as BWP17 is originally derived from SC5314, they may nevertheless have become genetically different by transformation over time. Thus these differences in parent strains could explain the observed discrepancies between the phenotypes of the $\operatorname{Tn} 7$ 


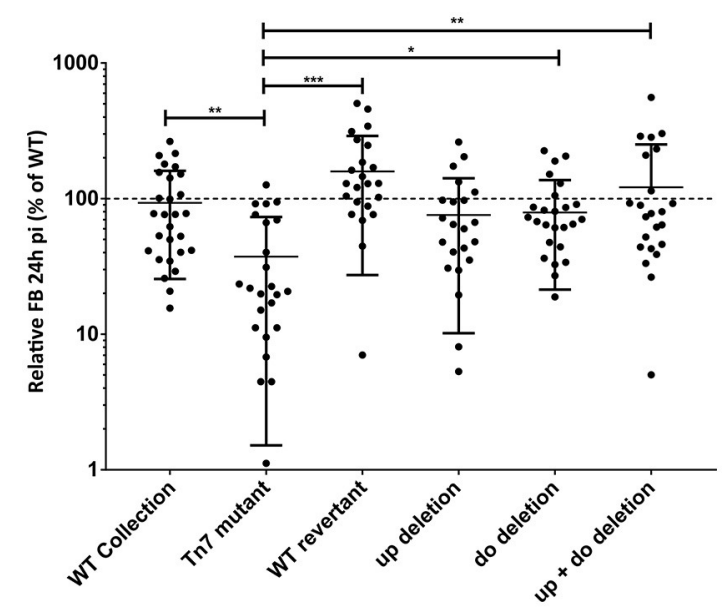

FIGURE 7 | Deletion of the Tn7 upstream and downstream regions and consequence on larvae fungal burden (FB). For each individual larva, the $\mathrm{FB}$ is expressed as a percentage relative to the mean of the wild-type group in the same experiment. All experiments were pooled in this graph. The mean value of each group is indicated with a black bar. The dotted line indicates the level of the mean of the wild-type strain set at $100 \%$. Each mutant was tested at least twice using 10 larvae each time and once using 5 larvae (missing dots correspond to dead larvae). Statistical analyses were performed using a ROUT analysis to remove outliers (Motulsky and Brown, 2006), followed by a Kruskal-wallis (non-parametric ANOVA) analysis with a Dunn post-test to assess CFU differences relative to the wild-type strain (GraphPad Prism 7.02) The stars indicate the level of statistical significance: ${ }^{*} p<0.05$, ${ }^{* *} p<0.01$, ${ }^{* * *} p<0.001$

mutant and of the up + do revertant. No difference in sequence was detected in the promoter regions of orf19.2646 in the BWP17 and SC5314 strains or in the Tn7 interruption mutant (data not shown). Therefore, the differences in the expression levels do not depend on polymorphism in the promoter region, but rather on another difference between these two strains.

Another explanation for the difference in fungal burden phenotypes might come from the URA3 marker positioning. The auxotrophic marker $U R A 3$ is known to play a role in the fungus colonization of its host (Brand et al., 2004). The two transcripts possess a wild-type copy of URA3 that is located at its original locus, while in the Tn7 interruption mutant, URA3 is located within orf19.2646 genomic locus. This factor could therefore play a role in the G. mellonella fungal burden level in addition to the differences of mRNA expression.

In this study we describe how the use of the $\operatorname{Tn} 7$ transposon for mutagenesis could lead to artifacts in C. albicans as has been described previously for filamentous fungi (Lo et al., 2003), $S$. cerevisiae (de Jesus Ferreira et al., 2001), and C. glabrata (Borah et al., 2011). Indeed, we show that the $\operatorname{Tn} 7$ transposon promotes a premature polyadenylation of the interrupted gene. Surprisingly and for the first time, we also identified the presence a $\operatorname{Tn} 7$ downstream transcript. In this last case, the Tn7 sequence might play the role of a transcriptional activator. In consequence, the phenotypic effects observed in the screening are not due to the absence of the gene of interest but are rather due to the existence of chimeric transcripts.

One of the options to avoid such an artifact would be to screen at least 2 or 3 Tn7 mutants of the same gene and consider it a "stable" phenotype if all mutants display the same phenotype. This would reduce the number of false positives for a given phenotype. However, these insertion mutants may display different phenotypes to the deletion mutants due to the presence of truncated protein expression, and might reveal unsuspected cellular process implicated in the phenotype studied. In addition, transposon insertions might occur in an essential gene as described in a C. glabrata study (Borah et al., 2011) and may reveal functions of truncated versions of the gene. Finally the Tn7 might be inserted in predicted non-coding region but may display a phenotype, thus revealing non-annotated loci (de Jesus Ferreira et al., 2001).

In the current study, the two unexpected transcripts interfere with a biological pathway involved in virulence or at least in adaptation to the host. In the future, tagging these transcripts will allow us to confirm their translation into proteins and to visualize them within the cell. Further transcriptional analysis would allow us to understand which pathways are modulated by expression of those new proteins. All together this study identified two unexpected transcripts involved in reduced fungal burden. This opened new avenue of investigation on a yet unidentified virulence mechanism involved in the host invasion process.

\section{AUTHOR CONTRIBUTIONS}

All authors were involved in the design of the studies and in the interpretation of the data. AP and FI carried out the majority of the experimental work, especially molecular biology experiments under the supervision of AC. AP and AC carried out the Galleria experiments. In addition to its involvement in the design of the studies, AC provided background information, strains and a critical input into the preparation of the manuscript. AP and AC wrote the manuscript.

\section{FUNDING}

This project was supported by the Swiss National Science Foundation through grant number PMPDP3_13960 and by the Novartis Science foundation.

\section{ACKNOWLEDGMENTS}

Thanks to Pr. Dominique Sanglard for his scientific support and his critical reading of the manuscript. Thanks to Eric Delarze for his critical reading of the manuscript and the figures.

\section{SUPPLEMENTARY MATERIAL}

The Supplementary Material for this article can be found online at: http://journal.frontiersin.org/article/10.3389/fmicb. 2017.00873/full\#supplementary-material 


\section{REFERENCES}

Akers, K. S., Cardile, A. P., Wenke, J. C., and Murray, C. K. (2015). Biofilm formation by clinical isolates and its relevance to clinical infections. Adv. Exp. Med. Biol. 830, 1-28. doi: 10.1007/978-3-319-11038-7_1

Amorim-Vaz, S., Delarze, E., Ischer, F., Sanglard, D., and Coste, A. T. (2015). Examining the virulence of Candida albicans transcription factor mutants using Galleria mellonella and mouse infection models. Front. Microbiol. 6:367. doi: 10.3389/fmicb.2015.00367

Basehoar, A. D., Zanton, S. J., and Pugh, B. F. (2004). Identification and distinct regulation of yeast TATA box-containing genes. Cell 116, 699-709. doi: 10.1016/S0092-8674(04)00205-3

Borah, S., Shivarathri, R., and Kaur, R. (2011). The RhoGAP CgBem2 is required for survival of azole stress in Candida glabrata. J. Biol. Chem. 286, 34311-34324. doi: 10.1074/jbc.M111.264671

Brand, A., MacCallum, D. M., Brown, A. J., Gow, N. A., and Odds, F. C. (2004). Ectopic expression of URA3 can influence the virulence phenotypes and proteome of Candida albicans but can be overcome by targeted reintegration of URA3 at the RPS10 locus. Eukaryot. Cell 3, 900-909. doi: 10.1128/EC.3.4.900-909.2004

Braun, B. R., and Johnson, A. D. (2000). TUP1, CPH1 and EFGal make independent contributions to filamentation in Candida albicans. Genetics 155, 57-67.

Chamilos, G., Nobile, C. J., Bruno, V. M., Lewis, R. E., Mitchell, A. P., and Kontoyiannis, D. P. (2009). Candida albicans Cas5, a regulator of cell wall integrity, is required for virulence in murine and toll mutant fly models. J. Infect. Dis. 200, 152-157. doi: 10.1086/599363

Chen, C., and Noble, S. M. (2012). Post-transcriptional regulation of the Sef1 transcription factor controls the virulence of Candida albicans in its mammalian host. PLoS Pathog. 8:e1002956. doi: 10.1371/journal.ppat.1002956

Chen, C., Pande, K., French, S. D., Tuch, B. B., and Noble, S. M. (2011). An iron homeostasis regulatory circuit with reciprocal roles in Candida albicans commensalism and pathogenesis. Cell Host Microbe 10, 118-135. doi: 10.1016/j.chom.2011.07.005

de Jesus Ferreira, M. C., Bao, X., Laize, V., and Hohmann, S. (2001). Transposon mutagenesis reveals novel loci affecting tolerance to salt stress and growth at low temperature. Curr. Genet. 40, 27-39. doi: 10.1007/s002940100237

Fuchs, B., Eby, J., Nobile, C., El Khoury, J., Mitchell, A., and Mylonakis, E. (2010). Role of filamentation in Galleria mellonella killing by Candida albicans. Microbes Infect. 12, 488-496. doi: 10.1016/j.micinf.2010.03.001

Gudlaugsson, O., Gillespie, S., Lee, K., Vande Berg, J., Hu, J., Messer, S., et al. (2003). Attributable mortality of nosocomial candidemia, revisited. Clin. Infect. Dis. 37, 1172-1177. doi: 10.1086/378745

Harriott, M. M., and Noverr, M. C. (2011). Importance of Candidabacterial polymicrobial biofilms in disease. Trends Microbiol. 19, 557-563. doi: 10.1016/j.tim.2011.07.004

Klein, B. S., and Tebbets, B. (2007). Dimorphism and virulence in fungi. Curr. Opin. Microbiol. 10, 314-319. doi: 10.1016/j.mib.2007.04.002

Lo, C., Adachi, K., Shuster, J. R., Hamer, J. E., and Hamer, L. (2003). The bacterial transposon Tn7 causes premature polyadenylation of mRNA in eukaryotic organisms: TAGKO mutagenesis in filamentous fungi. Nucleic Acids Res. 31, 4822-4827. doi: 10.1093/nar/gkg676

Lortholary, O., Renaudat, C., Sitbon, K., Madec, Y., Denoeud-Ndam, L., Wolff, M., et al. (2014). Worrisome trends in incidence and mortality of candidemia in intensive care units (Paris area, 2002-2010). Intensive Care Med. 40, 1303-1312. doi: $10.1007 /$ s00134-014-3408-3
McNeil, M. M., Nash, S. L., Hajjeh, R. A., Phelan, M. A., Conn, L. A., Plikaytis, B. D., et al. (2001). Trends in mortality due to invasive mycotic diseases in the United States, 1980-1997. Clin. Infect. Dis. 33, 641-647. doi: 10.1086/322606

Moqtaderi, Z., Geisberg, J. V., Jin, Y., Fan, X., and Struhl, K. (2013). Species-specific factors mediate extensive heterogeneity of mRNA $3^{\prime}$ ends in yeasts. Proc. Natl. Acad. Sci. U.S.A. 110, 11073-11078. doi: 10.1073/pnas.1309384110

Motulsky, H. J., and Brown, R. E. (2006). Detecting outliers when fitting data with nonlinear regression - a new method based on robust nonlinear regression and the false discovery rate. BMC Bioinformatics 7:123. doi: 10.1186/1471-2105-7-123

Nobile, C. J., and Johnson, A. D. (2015). Candida albicans Biofilms and human disease. Annu. Rev. Microbiol. 69, 71-92. doi: 10.1146/annurev-micro-091014-104330

Nobile, C., and Mitchell, A. (2009). Large-scale gene disruption using the UAU1 cassette. Methods Mol. Biol. 499, 175-194. doi: 10.1007/978-1-60327-151-6_17

Noble, S. M. (2013). Candida albicans specializations for iron homeostasis: from commensalism to virulence. Curr. Opin. Microbiol. 16, 708-715. doi: 10.1016/j.mib.2013.09.006

Puig-Asensio, M., Padilla, B., Garnacho-Montero, J., Zaragoza, O., Aguado, J. M., Zaragoza, R., et al. (2014). Epidemiology and predictive factors for early and late mortality in Candida bloodstream infections: a population-based surveillance in Spain. Clin. Microbiol. Infect. 20, O245-O254. doi: 10.1111/1469-0691.12380

Rajendran, R., Sherry, L., Nile, C. J., Sherriff, A., Johnson, E. M., Hanson, M. F., et al. (2016). Biofilm formation is a risk factor for mortality in patients with Candida albicans bloodstream infection-Scotland, 2012-2013. Clin. Microbiol. Infect. 22, 87-93. doi: 10.1016/j.cmi.2015.09.018

Reuss, O., Vik, A., Kolter, R., and Morschhauser, J. (2004). The SAT1 flipper, an optimized tool for gene disruption in Candida albicans. Gene 341, 119-127. doi: 10.1016/j.gene.2004.06.021

Sanglard, D., Ischer, F., Monod, M., and Bille, J. (1996). Susceptibilities of Candida albicans multidrug transporter mutants to various antifungal agents and other metabolic inhibitors. Antimicrob. Agents Chemother. 40, 2300-2305.

Sierro, F., Dubois, B., Coste, A., Kaiserlian, D., Kraehenbuhl, J. P., and Sirard, J. C. (2001). Flagellin stimulation of intestinal epithelial cells triggers CCL20mediated migration of dendritic cells. Proc. Natl. Acad. Sci. U.S.A. 98, 13722-13727. doi: 10.1073/pnas.241308598

Vandeputte, P., Ischer, F., Sanglard, D., and Coste, A. T. (2011). In vivo systematic analysis of Candida albicans Zn2-Cys6 transcription factors mutants for mice organ colonization. PLOS ONE 6:e26962. doi: 10.1371/journal.pone.00 26962

Wisplinghoff, H., Ebbers, J., Geurtz, L., Stefanik, D., Major, Y., Edmond, M. B., et al. (2014). Nosocomial bloodstream infections due to Candida spp. in the USA: species distribution, clinical features and antifungal susceptibilities. Int. J. Antimicrob. Agents 43, 78-81. doi: 10.1016/j.ijantimicag.2013. 09.005

Conflict of Interest Statement: The authors declare that the research was conducted in the absence of any commercial or financial relationships that could be construed as a potential conflict of interest.

Copyright (c) 2017 Pierrehumbert, Ischer and Coste. This is an open-access article distributed under the terms of the Creative Commons Attribution License (CC BY). The use, distribution or reproduction in other forums is permitted, provided the original author(s) or licensor are credited and that the original publication in this journal is cited, in accordance with accepted academic practice. No use, distribution or reproduction is permitted which does not comply with these terms. 